\title{
HYPERSPECTRAL DATA CLASSIFICATION USING FACTOR GRAPHS
}

\author{
Aliaksei Makarau, Rupert Müller, Gintautas Palubinskas, and Peter Reinartz \\ German Aerospace Center (DLR) \\ German Remote Sensing Data Center (DFD) bzw. Remote Sensing Technology Institute (IMF) \\ 82234 Oberpfaffenhofen, Germany \\ \{aliaksei.makarau, rupert.mueller, gintautas.palubinskas, peter.reinartz\}@dlr.de
}

Commission VII/3

KEY WORDS: Hyper spectral, Classification, Training, Reference Data

\begin{abstract}
:
Accurate classification of hyperspectral data is still a competitive task and new classification methods are developed to achieve desired tasks of hyperspectral data use. The objective of this paper is to develop a new method for hyperspectral data classification ensuring the classification model properties like transferability, generalization, probabilistic interpretation, etc. While factor graphs (undirected graphical models) are unfortunately not widely employed in remote sensing tasks, these models possess important properties such as representation of complex systems to model estimation/decision making tasks.

In this paper we present a new method for hyperspectral data classification using factor graphs. Factor graph (a bipartite graph consisting of variables and factor vertices) allows factorization of a more complex function leading to definition of variables (employed to store input data), latent variables (allow to bridge abstract class to data), and factors (defining prior probabilities for spectral features and abstract classes; input data mapping to spectral features mixture and further bridging of the mixture to an abstract class). Latent variables play an important role by defining two-level mapping of the input spectral features to a class. Configuration (learning) on training data of the model allows calculating a parameter set for the model to bridge the input data to a class.

The classification algorithm is as follows. Spectral bands are separately pre-processed (unsupervised clustering is used) to be defined on a finite domain (alphabet) leading to a representation of the data on multinomial distribution. The represented hyperspectral data is used as input evidence (evidence vector is selected pixelwise) in a configured factor graph and an inference is run resulting in the posterior probability. Variational inference (Mean field) allows to obtain plausible results with a low calculation time. Calculating the posterior probability for each class and comparison of the probabilities leads to classification. Since the factor graphs operate on input data represented on an alphabet (the represented data transferred into multinomial distribution) the number of training samples can be relatively low.

Classification assessment on Salinas hyperspectral data benchmark allowed to obtain a competitive accuracy of classification. Employment of training data consisting of 20 randomly selected points for a class allowed to obtain the overall classification accuracy equal to $85.32 \%$ and Kappa equal to 0.8358 . Representation of input data on a finite domain discards the curse of dimensionality problem allowing to use large hyperspectral data with a moderately high number of bands.
\end{abstract}

\section{INTRODUCTION}

Development of new methods for single/multisensory data classification leads to an improvement of the data classification and a more precise identification of land-cover classes. Nevertheless, requirements on the methods such as transferability, integration into complex systems, or augmenting ability motivate an employment of probabilistic graphical models [Bishop, 2006]. Application of probabilistic graphical models becomes more and more popular and efficient solution for image annotation, classification, for definition of semantic link between data and a high level labels [Lienou et al., 2010], [Bratasanu et al., 2011], [Wang et al., 2009].

Factor graphs (FG) were proposed in 1997 [Kschischang et al., 2001] and since then the application of FGs for signal/image processing and recognition is gradually emerging. B. Frey et al. [Frey and Jojic, 2005] performed a work on a comparison of learning and inference methods for probabilistic graphical models (Bayesian networks, Markov random fields, factor graphs). Factor graph is a convenient tool to define complex systems for data processing/interpretation, to expand the systems, allow to model complex interactions among a system parts (e.g. map features/properties from low to high level), to perform approximate inference on data, or use non full data for plausible decision making. Nevertheless, application of factor graphs as a more general graphical model type is not so wide for remotely sensed data interpretation.

In this paper a new approach for hyperspectral imagery supervised classification using factor graph is proposed. The structure of the factor graph is defined in order to define prior probabilities for input data, to map the input data to a latent variable (a mixture of the input features) and bridge the mixture to a semantic class. A configuration of the factor graph on training data allows to estimate the parameter set of the graph (probabilistic functions in the factors) and an employment of a fast inference method (Mean field [Frey and Jojic, 2005]) allows to obtain a competitive accuracy of the hyperspectral data classification.

\section{FACTOR GRAPH MODEL FOR CLASSIFICATION}

Factor graph (undirected probabilistic model) is a more general graphical model than a Bayesian network or a Markov random field. An FG possesses properties of Bayesian network and Markov random field and allows to describe complex relationships among parts of a modeled system. A factor graph is a bipartite graph containing two types of nodes: variable nodes $\left(x_{i}, i=1 . . n\right)$ and function nodes (factors) $\left(f_{j}\left(x_{1}, x_{2}, \ldots, x_{n}\right), j=1 . . m\right)$, where a variable node $x_{i}$ takes value on a finite domain (alphabet $A_{i}$ ) [Kschischang et al., 2001]. Figure 1 presents an example of 
a factor graph with three variables $x_{1}, x_{2}, x_{3}$ and two function nodes $f_{1}$ and $f_{2}$ with factorization: $g\left(x_{1}, x_{2}, x_{3}\right)=f_{1}\left(x_{1}, x_{2}\right)$ * $f_{2}\left(x_{2}, x_{3}\right)$.

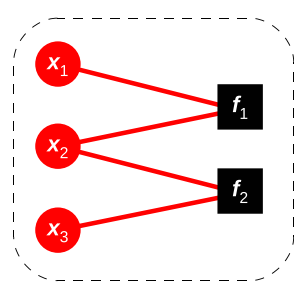

Figure 1: An illustrative example of simple factor graph with three variables $x_{1}, x_{2}, x_{3}$ and two function nodes $f_{1}\left(x_{1}, x_{2}\right)$ and $f_{2}\left(x_{2}, x_{3}\right)$.

The task of classification consists of determining the probability of a particular hypothesis given some observed evidence. This is solved by calculation of marginal probability of a latent variable, or by calculating of the maximum likelihood probability (maximum likelihood on the configured factor graph given the evidence). The likelihood of the evidence (the features vector) can be written as follows:

$$
p\left(\boldsymbol{x}, c \mid s_{k}\right)=\prod_{k=1}^{K} \prod_{n=1}^{N} p\left(x_{n} \mid c_{k}\right) p\left(c_{k} \mid s_{k}\right),
$$

where $\boldsymbol{x}$ is the input evidence; $s_{k}$ is the class; $c_{k}$ is the features mixture (a latent variable); $K$ is the number of classes; $N$ is the number of features in the input evidence vector. Here, the feature is assumed to be a spectral band value.

The following factor graph structure can be defined for formula (1):

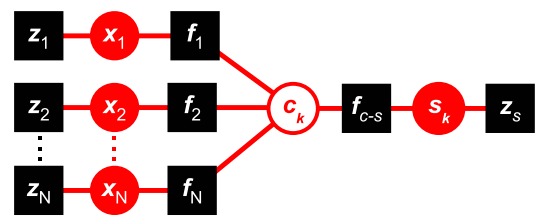

Figure 2: Factor graph model (independent model; a mixture latent variable is employed) for hyperspectral data classification

The factor graph is described as:

$$
\begin{array}{r}
g\left(x_{1}, x_{2}, \ldots, x_{N}, c_{k}, s_{s}\right)=\prod_{n=1}^{N} z_{n}\left(x_{n}\right) \prod_{n=1}^{N} f_{n}\left(x_{n}, c_{k}\right) \\
f_{c-s}\left(c_{k}, s_{k}\right) z_{s}\left(s_{k}\right),
\end{array}
$$

where $x_{n}$ is the $n$-th input feature; $c_{k}$ is the features mixture (a latent variable) for the $k$-th class; $s_{k}$ is the $k$-th class number; $z_{1}, \ldots, z_{N}, z_{s}$ are normalizing factors in the graph model [Frey and Jojic, 2005] (define prior probabilities); $f_{1}, \ldots, f_{N}$ are the factors mapping the input features to the feature mixture; $f_{c-s}$ is the factor bridging the latent variable to the semantic class $s_{k}$.

The structure of a factor graph defines a dependency of class variable node on input features. Use of training data allows to calculate a configuration (parameter set $\theta(k)$ ) for a factor graph for each class $k$.

A configured factor graph with the configuration $\theta(k)$ is expected to have a maximum likelihood probability (a low energy state) on the evidence which most likely (similar) to the employed training data. Expectation maximization method with gradient ascent optimizer are employed for learning the graph configuration. Mean field inference method [Frey and Jojic, 2005] is employed for the inference. Comparison of the classes probability maps (maximum principle) allows to produce label map.

\subsection{Employed data}

Salinas hyperspectral data benchmark (AVIRIS sensor over Salinas Valley, California; $3.7 \mathrm{~m}$ pixel size) was selected for classification accuracy evaluation. The data cube size is 512 lines by 217 samples, 224 bands. 19 water absorption bands were discarded (bands [108-112] and [154-167]) This image is available as at-sensor radiance data. Ground truth classes and the number of samples are given in Table 1 .

Minimum noise fraction (MNF) [Green et al., 1988, Boardman and Kruse, 194] was employed to reduce the number of input features, reduce computational time, and separate noise from the data. The MNF consists of two Principal Components (PC) transformations. The first PC transformation decorrelates and rescales noise in the data, the second PC transformation performed on the noise-cleared data.

Since factor graphs are discrete graphical models, the feature is to be represented on a predefined finite domain (alphabet). The finite domain refers to the unique values (or a list of values) the feature can have. Here we use a finite domain consisting of natural numbers. To represent an input feature on the finite domain, the feature is proposed to be processed separately by an unsupervised clustering. A cluster's number is assumed as the value from the defined domain. $k$-means procedure is employed and the number of clusters for feature representation on finite set was equal to 100 (used for representation of all features). In an additional experiment, the features before representation are processed by median filtering.

20 points were randomly selected for each class in order to configure the factor graph. Expectation maximization with a gradient ascent method were employed for the factor graph configuration.

Table 1: Salinas hyperspectral benchmark classes (available ground truth samples)

\begin{tabular}{|c|c|c|}
\hline Number & Class & Samples \\
\hline \hline 1 & Brocoli_green_weeds_1 & 2009 \\
\hline 2 & Brocoli_green_weeds_2 & 3726 \\
\hline 3 & Fallow & 1976 \\
\hline 4 & Fallow_rough_plow & 1394 \\
\hline 5 & Stubble & 3959 \\
\hline 6 & Celery & 3579 \\
\hline 7 & Grapes_untrained & 11271 \\
\hline 8 & Soil_vinyard_develop & 6203 \\
\hline 9 & Corn_senesced_green_weeds & 3278 \\
\hline 10 & Lettuce_romaine_4wk & 1068 \\
\hline 11 & Lettuce_romaine_5wk & 1927 \\
\hline 12 & Lettuce_romaine_6wk & 916 \\
\hline 13 & Lettuce_romaine_7wk & 1070 \\
\hline 14 & Vinyard_untrained & 7268 \\
\hline 15 & Fallow_smooth & 2678 \\
\hline 16 & Vinyard_vertical_trellis & 1807 \\
\hline
\end{tabular}

\section{RESULTS AND DISCUSSION}

Table 2 presents the overall accuracy and Kappa coefficient for the classification. The additional experiment with feature median filtering allowed to obtain a better classification accuracy results (overall accuracy $=85.3217$ and Kappa $=0.8358$ versus 81.3692 and 0.7921 , respectively; compare confusion Tables 3 and 4). Most of the classes were labeled with an accuracy more than $90 \%$, 
except several most difficult classes (these classes are usually hard to label and are mainly confused, see Tables 3 and 4). The classes 7 (Grapes_untrained) and 14 (Vinyard_untrained) illustrate the highest confusion with classification accuracies equal to $68.79 \%$ and $65.05 \%$ (Table 4 ), respectively. Classes 11 (Lettuce_romaine_5wk) and 8 (Soil_vinyard_develop), also class 13 (Lettuce_romaine_7wk) and 12 (Lettuce_romaine_6wk) and 11 (Lettuce_romaine_5wk) are less confused.

Median filtering reduced the influence of the outlier samples in the input data for classification. Confusion among classes was reduced and a better labeling was reached. MNF data preprocessing allows to reduce the time of calculation with a competitive classification accuracy. On full bands set data a better classification accuracy is expected to be obtained.

Approximate inference methods should be employed for the likelihood probability computation. Approximate inference allows to calculate decisions with the accuracy comparable to the results of full propagation methods but with a high reduction of run time. In this work Mean Field approximate inference method was employed. Factor graph allows to perform an inference for one class (to produce a probability map) leading to an application of material detection in hyperspectral data.

Among disadvantages we can note that probabilistic models require computational time higher than many classification methods, since inference in each point of input data is performed. Also maximum principle on the likelihood probability maps (performed to obtain class label map) can be a source of misclassification.

Table 2: The accuracy of salinas benchmark classification using the FG (MNF 20 features, alphabet size: 100). Additional experiment with feature median filtering is also presented. OVA overall accuracy, Kappa - Cohen's Kappa

\begin{tabular}{|l|c|c|}
\hline Method & OVA, \% & Kappa \\
\hline FG & 81.3692 & 0.7921 \\
\hline FG $($ Median $5 \times 5)$ & 85.3217 & 0.8358 \\
\hline
\end{tabular}

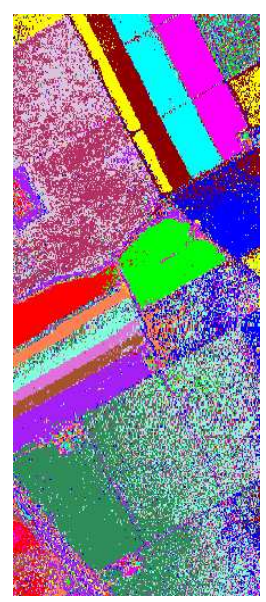

(a)

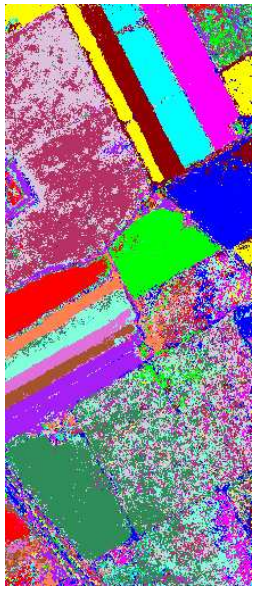

(b)

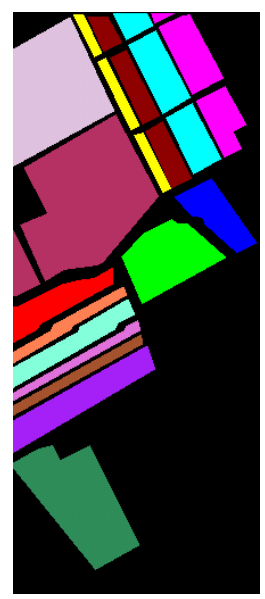

(c)
Figure 3: Classification maps for Salinas benchmark using factor graphs: (a) MNF, 20 features, alphabet size: 100, (b) MNF, 20 features, median filtering $5 \times 5$, alphabet size: 100 , (c) ground truth label map

\section{CONCLUSIONS}

The paper presents another successful area of factor graphs application: multispectral data classification. A relatively simple structure of the FG allows to reach a competitive accuracy of classification even on data with decreased radiometric range (represented on the alphabet). An important property of factor graph classification is that the method requires a relatively low number of training samples (only 20 points for a class). Separate processing of input features (spectral bands) and employment of the presented data fusion and classification model is not influenced by the limitations of data dimensionality (i.e. there is no the curse of dimensionality). Classification on full data (all spectral bands) is possible to run (comparing to MNF features) and will take more computational time.

\section{REFERENCES}

Bishop, C., 2006. Pattern recognition and machine learning. Information Science and Statistics. Springer, New York.

Boardman, J. and Kruse, F., 194. Automated spectral analysis: a geological example using aviris data, north grapevine mountains, nevada. In: Proceedings of the Tenth Thematic Conference on Geological Remote Sensing, San Antonio, TX, USA, pp. 407418.

Bratasanu, D., Nedelcu, I. and Datcu, M., 2011. Bridging the semantic gap for satellite image annotation and automatic mapping applications. IEEE Journal of Selected Topics in Applied Earth Observations and Remote Sensing 4(1), pp. 193-204.

Frey, B. and Jojic, N., 2005. A comparison of algorithms for inference and learning in probabilistic graphical models. IEEE Transactions on Pattern Analysis and Machine Intelligence 27(9), pp. 1392-1416.

Green, A., Berman, M., Switzer, P. and Craig, M., 1988. A transformation for ordering multispectral data in terms of image quality with implications for noise removal. IEEE Transactions on Geoscience and Remote Sensing 26(1), pp. 65-74.

Kschischang, F., Frey, B. and Loeliger, H.-A., 2001. Factor graphs and the sum-product algorithm. IEEE Transactions on Information Theory 47(2), pp. 498-519.

Lienou, M., Maitre, H. and Datcu, M., 2010. Semantic annotation of satellite images using latent Dirichlet allocation. IEEE Geoscience and Remote Sensing Letters 7(1), pp. 28-32.

Wang, C., Blei, D. and Fei-Fei, L., 2009. Simultaneous image classification and annotation. In: IEEE Conference on Computer Vision and Pattern Recognition, pp. 1903-1910. 
Table 3: Confusion matrix of the FG classification on Salinas benchmark (MNF 20 features, alphabet size: 100). Overall accuracy $=81.3692$, Kappa=0.7921 Percentages are given for an easier interpretation.

\begin{tabular}{|c|c|c|c|c|c|c|c|c|c|c|c|c|c|c|c|}
\hline & \multicolumn{15}{|c|}{ Ground truth, see Table 1} \\
\hline Class & 1 & 2 & 3 & 4 & 5 & 6 & 7 & 8 & 9 & 10 & 11 & 12 & 13 & 14 & 15 \\
\hline 1 & 98.36 & 0.48 & 0.00 & 0.00 & 0.03 & 0.06 & 0.13 & 0.00 & 0.03 & 0.00 & 0.00 & 0.00 & 0.00 & 0.00 & 0.00 \\
\hline 2 & 1.14 & 96.54 & 0.00 & 0.00 & 0.00 & 0.00 & 0.15 & 0.00 & 0.34 & 0.00 & 0.00 & 0.00 & 0.00 & 0.01 & 0.00 \\
\hline 3 & 0.00 & 0.05 & 87.96 & 1.51 & 0.38 & 0.11 & 0.90 & 0.56 & 1.07 & 0.28 & 1.19 & 0.00 & 0.00 & 0.34 & 0.78 \\
\hline 4 & 0.00 & 0.00 & 0.35 & 94.62 & 0.03 & 0.00 & 0.20 & 0.02 & 0.12 & 0.19 & 0.42 & 0.00 & 0.00 & 0.07 & 2.43 \\
\hline 5 & 0.20 & 0.03 & 0.00 & 0.00 & 96.36 & 0.00 & 0.09 & 0.02 & 0.15 & 0.09 & 0.47 & 0.00 & 0.00 & 0.01 & 0.15 \\
\hline 6 & 0.05 & 1.58 & 0.00 & 0.14 & 0.08 & 99.58 & 0.52 & 0.10 & 0.12 & 0.28 & 0.05 & 0.00 & 0.47 & 0.26 & 0.11 \\
\hline 7 & 0.15 & 0.03 & 0.30 & $\overline{0.00}$ & 0.20 & 0.03 & 58.73 & 0.32 & 0.24 & 0.28 & 0.99 & 0.00 & 0.09 & 35.84 & 0.00 \\
\hline 8 & 0.05 & 0.05 & 0.46 & 0.22 & 0.33 & 0.06 & $\overline{\overline{1.61}}$ & 95.70 & 2.10 & 3.56 & 14.58 & 0.33 & 0.09 & 1.33 & 0.07 \\
\hline 9 & 0.00 & 0.03 & 0.15 & 0.22 & 0.20 & 0.03 & 2.32 & 0.55 & 91.00 & 2.06 & 3.94 & 0.00 & 1.31 & 1.31 & 0.11 \\
\hline 10 & 0.00 & 0.21 & 0.10 & 0.00 & 0.00 & 0.03 & 0.02 & 0.05 & 1.80 & 91.29 & 3.79 & 0.00 & 0.37 & 0.01 & 0.04 \\
\hline 11 & 0.05 & 0.05 & 0.05 & 0.00 & 0.51 & 0.00 & 1.54 & 1.79 & 0.98 & 1.31 & 70.37 & 3.93 & 2.06 & 0.39 & 0.00 \\
\hline 12 & 0.00 & 0.21 & 0.00 & 0.00 & 0.03 & 0.00 & 0.31 & 0.03 & 0.18 & 0.28 & $\overline{\overline{1.76}}$ & 93.45 & 7.57 & 0.03 & 0.00 \\
\hline 13 & 0.00 & 0.30 & 0.00 & 0.00 & 0.08 & 0.00 & 0.03 & 0.00 & 0.46 & 0.00 & 0.05 & 1.97 & 86.54 & 0.00 & 0.22 \\
\hline 14 & 0.00 & 0.38 & 0.71 & 0.07 & 0.91 & 0.11 & 32.80 & 0.77 & 1.19 & 0.37 & 2.18 & 0.33 & 1.31 & 59.87 & 0.37 \\
\hline 15 & $\overline{0.00}$ & 0.05 & 9.92 & 3.23 & 0.88 & 0.00 & 0.64 & 0.10 & 0.21 & 0.00 & 0.21 & 0.00 & 0.19 & $\overline{0.52}$ & 95.71 \\
\hline
\end{tabular}

Table 4: Confusion matrix of the FG classification on Salinas benchmark (MNF 20 features, alphabet size: 100, feature median $5 \times 5$ filtering). Overall accuracy $=85.3217$, Kappa $=0.8358$ Percentages are given for an easier interpretation.

\begin{tabular}{|c|c|c|c|c|c|c|c|c|c|c|c|c|c|c|c|c|}
\hline & \multicolumn{10}{|c|}{ Ground truth, see Table 1 } \\
\hline Class & 1 & 2 & 3 & 4 & 5 & 6 & 7 & 8 & 9 & 10 & 11 & 12 & 13 & 14 & 15 \\
\hline 1 & 98.90 & 0.81 & 0.00 & 0.00 & 0.10 & 0.00 & 0.23 & 0.00 & 0.00 & 0.00 & 0.00 & 0.00 & 0.19 & 0.00 & 0.00 \\
\hline 2 & 0.25 & 96.81 & 0.00 & 0.00 & 0.10 & 0.00 & 0.31 & 0.02 & 0.85 & 0.00 & 0.00 & 0.00 & 0.09 & 0.11 & 0.00 \\
\hline 3 & 0.00 & 0.05 & 98.38 & 0.43 & 0.66 & 0.14 & 0.48 & 0.16 & 1.34 & 0.19 & 0.16 & 0.00 & 0.09 & 0.15 & 0.56 \\
\hline 4 & 0.00 & 0.03 & 0.00 & 96.99 & 0.00 & 0.06 & 0.07 & 0.00 & 0.06 & 0.00 & 0.00 & 0.00 & 0.00 & 0.10 & 2.05 \\
\hline 5 & 0.40 & 0.43 & 0.00 & 0.00 & 94.04 & 0.00 & 0.02 & 0.19 & 0.09 & 0.37 & 1.19 & 0.00 & 0.09 & 0.04 & 0.00 \\
\hline 6 & 0.05 & 0.56 & 0.00 & 0.00 & 0.05 & 99.66 & 0.78 & 0.34 & 0.18 & 0.00 & 0.10 & 0.00 & 1.03 & 0.37 & 0.34 \\
\hline 7 & 0.30 & 0.59 & 0.05 & 0.36 & 1.11 & 0.00 & 68.79 & 0.77 & 0.49 & 0.19 & 1.09 & 0.00 & 0.37 & 32.47 & 0.04 \\
\hline 8 & 0.00 & 0.16 & 0.10 & 0.50 & 0.48 & 0.03 & 1.46 & 96.08 & 0.92 & 1.31 & 10.33 & 0.00 & 0.28 & 1.28 & 0.00 \\
\hline 9 & 0.00 & 0.00 & 0.10 & 0.00 & 0.08 & 0.03 & 1.49 & 0.11 & 91.79 & 0.37 & 0.62 & 0.00 & 0.65 & 0.00 & 0.00 \\
\hline 10 & 0.00 & 0.00 & 0.00 & 0.00 & 0.00 & 0.00 & 0.04 & 0.00 & 0.58 & 96.25 & 1.19 & 0.00 & 0.00 & 0.00 & 0.00 \\
\hline 11 & 0.00 & 0.21 & 0.00 & 0.00 & 0.91 & 0.03 & 0.83 & 0.98 & 1.37 & 0.47 & 83.60 & 3.38 & 4.86 & 0.14 & 0.00 \\
\hline 12 & 0.00 & 0.21 & 0.00 & 0.00 & 0.03 & 0.00 & 0.03 & 0.00 & 0.03 & 0.00 & 0.00 & 96.07 & 7.66 & 0.00 & 0.00 \\
\hline 13 & 0.00 & 0.08 & 0.00 & 0.00 & 0.00 & 0.00 & 0.01 & 0.00 & 0.00 & 0.00 & 0.05 & 0.55 & 84.49 & 0.00 & 0.26 \\
\hline 14 & 0.10 & 0.05 & 0.05 & 0.29 & 1.67 & 0.06 & 25.24 & 1.34 & 2.23 & 0.84 & 1.66 & 0.00 & 0.09 & 65.05 & 0.07 \\
\hline 15 & 0.00 & 0.00 & 1.32 & 1.43 & 0.78 & 0.00 & 0.24 & 0.00 & 0.06 & 0.00 & 0.00 & 0.00 & 0.09 & 0.29 & 96.68 \\
\hline
\end{tabular}

